\title{
Identification of the A293 (AVE1231) Binding Site in the Cardiac Two-Pore- Domain Potassium Channel TASK-1: a Common Low Affinity Antiarrhythmic Drug Binding Site
}

\author{
Felix Wiedmanna,b,c Aytug K. Kiper ${ }^{d}$ Mauricio Bedoya Antonius Ratte ${ }^{a, c}$ \\ Susanne Rinnéd Manuel Krafta,b,c Maximilian Waibel ${ }^{a, b, c} \quad$ Priya Anad $^{f}$ \\ Wolfgang Wenzel ${ }^{f}$ Wendy González ${ }^{\mathrm{e}, g}$ Hugo A. Katus ${ }^{\mathrm{a}, \mathrm{b}, \mathrm{c}}$ Niels Decher ${ }^{\mathrm{d}}$ \\ Constanze Schmidta, $, \mathrm{b}, \mathrm{c}$
}

\begin{abstract}
aDepartment of Cardiology, University of Heidelberg, Heidelberg, Germany, ${ }^{b}$ DZHK (German Center for Cardiovascular Research), partner site Heidelberg /Mannheim, University of Heidelberg, Heidelberg, Germany, ' HCR, Heidelberg Center for Heart Rhythm Disorders, University of Heidelberg, Heidelberg, Germany, Institute for Physiology and Pathophysiology, Vegetative Physiology, University of Marburg, Marburg, Germany, ${ }^{e}$ Centro de Bioinformática y Simulación Molecular, Universidad de Talca, Talca, Chile, Institute of Nanotechnology, Karlsruhe Institute of Technology, Karlsruhe, Germany, 9Millennium Nucleus of Ion Channels-Associated Diseases (MiNICAD), Universidad de Talca, Talca, Chile
\end{abstract}

\section{Key Words}

A1899 • A293 • Atrial arrhythmopathy • Atrial cardiomyopathy • Atrial fibrillation • AVE1231 • Drug binding site $\cdot \mathrm{K}_{2 \mathrm{P}} 3.1 \cdot \mathrm{KCNK3} \cdot \mathrm{TASK}-1 \cdot$ Two-pore-domain potassium channels

\begin{abstract}
Background/Aims: The two-pore-domain potassium channel TASK-1 regulates atrial action potential duration. Due to the atrium-specific expression of TASK-1 in the human heart and the functional upregulation of TASK-1 currents in atrial fibrillation (AF), TASK-1 represents a promising target for the treatment of AF. Therefore, detailed knowledge of the molecular determinants of TASK- 1 inhibition may help to identify new drugs for the future therapy of AF. In the current study, the molecular determinants of TASK-1 inhibition by the potent and antiarrhythmic compound A293 (AVE1231) were studied in detail. Methods: Alanine-scanning mutagenesis together with two-electrode voltage-clamp recordings were combined with in silico docking experiments. Results: Here, we have identified Q126 located in the M2 segment together with L239 and N240 of the M4 segment as amino acids essential for the A293mediated inhibition of TASK-1. These data indicate a binding site which is different to that of

N. Decher and C. Schmidt contributed equally to this work.

\begin{tabular}{ll}
\hline PD Dr. med. Constanze & Medical University Hospital Heidelberg, Department of Cardiology, Im Neuenheimer Feld 410, \\
Schmidt & Heidelberg; Philipps-University Marburg, AG Vegetative Physiology; Deutschhausstraße 1-2, Marburg \\
and Prof. Dr. Niels Decher & (Germany), E-Mail Constanze.Schmidt@med.uni-heidelberg.de; Decher@staff.uni-marburg.de
\end{tabular}
\end{abstract}




\section{Cellular Physiology Cell Physiol Biochem 2019;52:1223-1235 \\ \begin{tabular}{ll|l} 
and Biochemistry $\begin{array}{l}\text { DOl: 10.33594/000000083 } \\
\text { Published online: } 19 \text { April 2019 }\end{array}$ & $\begin{array}{l}\text { O } 2019 \text { The Author(s). Published by } \\
\text { Cell Physiol Biochem Press GmbH\&Co. KG }\end{array}$ \\
\cline { 2 - 3 } &
\end{tabular} \\ Wiedmann et al.: TASK-1 Drug Binding Site of A293}

A1899 for which also residues of the pore signature sequence and the late M4 segments are essential. Using in silico docking experiments, we propose a binding site at the lower end of the cytosolic pore, located at the entry to lateral side fenestrations of TASK-1. Strikingly, TASK1 inhibition by the low affinity antiarrhythmic TASK-1 blockers propafenone, amiodarone and carvedilol was also strongly diminished by mutations at this novel binding site. Conclusion: We have identified the A293 binding site in the central cavity of TASK-1 and propose that this site might represent a conserved site of action for many low affinity antiarrhythmic TASK-1 blockers.

(C) 2019 The Author(s). Published by Cell Physiol Biochem Press GmbH\&Co. KG

\section{Introduction}

The family of two-pore-domain potassium $\left(\mathrm{K}_{2 \mathrm{p}}\right)$ channels is the last identified among potassium channels. The 15 members of this heterologous group share a unique structure of four membrane spanning domains and the eponymous two pore-forming loops per subunit [1]. Assembled as homo- or heterodimers, most $\mathrm{K}_{2 \mathrm{P}}$ channels conduct quasi-instantaneous, non-inactivating background potassium currents that modulate cellular excitability $[2,3] . \mathrm{K}_{2 \mathrm{P}}$ channels are regulated by a wide variety of physiological stimuli, including polyunsaturated fatty acids, mechanical stress, heat, $\mathrm{pH}$ level and pharmacologic agents [4]. Therefore, $\mathrm{K}_{2 \mathrm{P}}$ currents contribute to regulation of a large variety of physiological functions, including regulation of cardiac rhythm, blood pressure, exocrine secretion, sensation of oxygen tension, taste and temperature [5-9] Additionally, they are implicated in neuroprotection, anesthesia, apoptosis and carcinogenesis [10].

In the heart $\mathrm{K}_{2 \mathrm{P}} 3.1$ (TASK-1, tandem of $\mathrm{P}$ domains in a weak inward rectifying $\mathrm{K}^{+}$channel (TWIK)-related acid sensitive $\mathrm{K}^{+}$channel 1) $\mathrm{K}^{+}$currents contribute to the delayed rectifier sustained outward current $I_{\text {Ksus }}$ [11] observed in mouse cardiomyocytes or the plateau current $I_{\mathrm{KP}}[12]$, described in cardiomyocytes from guinea pig $[13,14]$. In the human heart, TASK-1 channels display virtually atrial specific expression [14] and inhibition of TASK-1 was shown to prolong action potential duration of atrial cardiomyocytes [14, 15]. Moreover, we could recently demonstrate that TASK-1 currents alter atrial action potential duration in patients suffering from atrial fibrillation (AF) or heart failure (HF) $[15,16]$.

$\mathrm{AF}$ is the most common sustained arrhythmia in clinical practice, constituting one of the major causes of stroke, acute HF, and cardiovascular morbidity [17]. Despite its epidemiological and individual relevance, safe and effective management of AF remains an unmet medical need as current pharmacological, interventional or surgical therapy strategies are limited by suboptimal effectiveness and not uncommonly by severe adverse effects [18]. Accordingly, there is a high medical need for novel drugs targeting AF.

Due to the very low ventricular expression levels of TASK-1 and the fact that TASK-1 inhibition prolonged human atrial action potential duration, TASK-1 currents represent a promising drug target for the treatment of AF [14]. This conclusion is further strengthened by recent observations, that in patients suffering from AF, TASK-1 currents are upregulated $[15,19]$, potentiating antiarrhythmic effects of a TASK-1 blockade. Additionally, a general role for TASK-1 channels as antiarrhythmic drug targets is supported by their sensitivity to clinically used antiarrhythmic agents like amiodarone, carvedilol, digoxin, dronedarone, mexiletine and propafenone when administered in supratherapeutic concentrations [9, 2022]. Molecular mechanisms of these interactions however remain far from clear. Until today only a few potent TASK-1 inhibitors are available.

However, first studies were already successfully exploring the role of TASK-1 channels utilizing these blockers, A293 and A1899, for isolation of native TASK-1 currents. Initially these compounds were developed as $\mathrm{K}_{\mathrm{v}} 1.5$ inhibitors by Sanofi-Aventis (Frankfurt, Germany), these experimental antiarrhythmic drugs later turned out to be much more potent TASK-1 inhibitors active in the nanomolar range [23]. Recently, the bis-amide ML365 was proposed as a further high affinity inhibitor of TASK-1 [24]. 


\section{Cellular Physiology Cell Physiol Biochem 2019;52:1223-1235 \\ \begin{tabular}{ll|l} 
and Biochemistry & $\begin{array}{l}\text { DOl: 10.33594/000000083 } \\
\text { Published online: } 19 \text { April } 2019\end{array}$ & $\begin{array}{l}\text { O } 2019 \text { The Author(s). Published by } \\
\text { Cell Physiol Biochem Press GmbH\&Co. KG }\end{array}$ \\
\cline { 2 - 3 } & Wedme
\end{tabular} \\ Wiedmann et al.: TASK-1 Drug Binding Site of A293}

A comprehensive analysis revealed a distinct set of amino acids that line the inner pore of TASK-1 to contribute to the block caused by A1899 (S20951) [25]. Whether these amino acids mediate TASK-1 interaction with other high affinity (e.g. A293, ML365) or low affinity blockers of TASK-1 remained unclear. Thus, we aimed to characterize the molecular interaction site of the potent blocker A293 with TASK-1 channels and to assess whether this drug binding site is also responsible for interactions with low affinity TASK-1 inhibitors like commonly used antiarrhythmic compounds.

\section{Materials and Methods}

\section{Molecular Biology}

Complementary DNAs encoding $\mathrm{hK}_{2 \mathrm{P}} 3.1$ (NM_002246) subcloned in the pRAT backbone (a dual purpose expression vector for in vitro RNA transcription and CMV promoter mediated expression in mammalian cells) were kindly provided by Steve Goldstein (Loyola University, Chicago, Il, USA). Alanine mutants of pRATTASK-1 were generated by site-directed mutagenesis, using the QuickChange II system (Agilent, Santa Clara, CA, USA), according to the manufacturer's instructions. Coding sequences of all constructs were completely sequenced (GATC-Biotech, Konstanz, Germany). In vitro synthesis of copy RNA was carried out, using the mMESSAGE mMACHINE T7 Transcription Kit (Thermo Fisher Scientific Waltham, MA, USA) according to the manufacturer's instructions. Nucleic acid concentration was determined by spectroscopy (ND-1000, Thermo Fisher Scientific) and integrity of transcripts was assessed via agarose gel electrophoresis.

\section{Oocyte Preparation}

Animal experiments have been carried out in accordance with the Guide for the Care and Use of Laboratory Animals as adopted and promulgated by the U.S. National Institutes of Health (NIH publication No. 86-23, revised 1985) and with EU Directive 2010/63/EU, and the current version of the German Law on the Protection of Animals was followed. The manuscript does not contain clinical studies or patient data.

The investigation conforms to the Directive 2010/63/EU of the European Parliament. Approval was granted by the local Animal Welfare Committee (reference number A-38/11). All experiments have been carried out in accordance with the United States National Institutes of Health Guide for the Care and Use of Laboratory Animals (NIH Publication No. 85-23, revised 1996) as reported earlier in detail [20, 21, 26]. Oocytes were isolated from Xenopus laevis ovarian lobes after surgical extirpation during tricaine anaesthesia ( $1 \mathrm{~g} / \mathrm{l}$; pH 7.5). Complementary RNA (0.75-18 ng; $46 \mathrm{nl} /$ oocyte) was injected into stage V-VI defolliculated Xenopus oocytes.

\section{Electrophysiology}

Two-electrode voltage clamp recordings from Xenopus laevis oocytes were performed one to three days after cRNA injection as described [27]. Macroscopic currents were measured with an OC-725C amplifier (Warner Instruments, Hamden, CT, USA). Electrodes were fabricated from borosilicate glass (GB 100F-10, Science Products, Hofheim, Germany) using a Flaming/Brown P-87 micropipette puller (Sutter Instruments, Novato, CA, USA) and had tip resistances from 1-2 MOhm. All experiments were carried out at room temperature $\left(20-22^{\circ} \mathrm{C}\right)$ under constant superfusion with extracellular solution by a gravity driven perfusion system. Data were filtered through an eight-pole Bessel filter and leak currents were not subtracted. The holding potential was $-80 \mathrm{mV}$ in all experiments.

\section{Solutions and Drugs}

Two-electrode voltage clamp electrodes were filled with $3 \mathrm{M} \mathrm{KCl}$. The standard extracellular solution for oocytes recordings contained $96 \mathrm{mM} \mathrm{NaCl}, 4 \mathrm{mM} \mathrm{KCl}, 1.1 \mathrm{mM} \mathrm{CaCl}$, 1 mM MgCl, $5 \mathrm{mM}$ 4-(2-hydroxyethyl)-1piperazineethanesulfonic acid (HEPES) (pH 7.4). The aromatic carbonamide A293 (2-(butylsulfonylamino)N-[(1R)-1-(6-methoxy-3-pyridyl) propyl]benzamide) was kindly provided by Sanofi-Aventis (Frankfurt, Germany). A $10 \mathrm{mM}$ DMSO stock of A293 was stored at $-20^{\circ} \mathrm{C}$. Amiodarone, carvedilol and propafenone were purchased from Sigma-Aldrich (Munich, Germany) and dissolved in DMSO at concentrations of $100 \mathrm{mM}\left(-20^{\circ} \mathrm{C}\right)$. On the day of experiments, aliquots of the stock solutions were diluted to the desired concentrations with standard extracellular solution. 


\section{Molecular modelling}

A TASK-1 homology model based on TWIK-1 channel (PDB ID: 3UMK) was used for docking calculations, as previously described [28]. The molecular docking calculation was performed using Glide v.7.4 with the standard precision (SP) mode [29]. Docking grid was centered on residues that significantly affects the conduction of the channel (Q126, L239, N240) and a cubic box with the axial length of 25A was used. The A293 ligand in its neutral state was prepared using LigPrep module (LigPrep, Schrödinger, LLC, New York, NY, USA, 2017). Energy minimization in the gas phase using Macromodel (MacroModel, Schrodinger, LLC, New York, NY, USA, 2017) with the OPLS2005 force field was performed. Twenty conformers of the ligand previously optimized were generated using ConfGen tool [30]. For the poses generation, it was enabled the strain correction for the GlideScore. Finally, one hundred poses were generated (five poses per conformer).

\section{Data Analysis, visualization and Statistics}

Current amplitudes were measured at the end of the $+20 \mathrm{mV}$ test pulse. Data are presented as mean \pm S.E.M. pClamp9 (Axon Instruments, Foster City, CA, USA) and Prism 5 (GraphPad, La Jolla, CA, USA) software was used for data acquisition and analysis. Statistical comparisons were performed using paired and unpaired Student's t tests (two-tailed tests), where appropriate. A P value $<0.05$ was considered statistically significant. Multiple comparisons were performed using the one-way ANOVA. If the hypothesis of equal means could be rejected at the 0.05-level, pair wise comparisons of groups were made and the probability values were adjusted for multiple comparisons using the Bonferroni correction. Visualizations of in silico simulations and dockings were generated with Pymol 2 (The PyMOL Molecular Graphics System, Version 2.0 Schrödinger, LLC, New York, NY, USA).

\section{Results}

TASK-1 inhibition by $A 293$

Inhibition of TASK-1 $\left(\mathrm{K}_{2 \mathrm{P}} 3.1\right)$ channels by the aromatic carbonamide A293 (AVE1231) (Fig. 1a) was studied after heterologous expression of the channel in Xenopus laevis oocytes. 24 - $48 \mathrm{~h}$ after injection of cRNA encoding human wild-type (WT) TASK-1, potassium currents were elicited by voltage steps to potentials ranging from -80 to $+60 \mathrm{mV}$ (500 ms), applied in $+20 \mathrm{mV}$ increments at a frequency of $0.2 \mathrm{~Hz}$. The holding potential was set to -80 $\mathrm{mV}$ and measurements were repeated every 2 minutes. Representative families of TASK- 1 current traces before and after application of $10 \mu \mathrm{M}$ A293 are depicted in Fig. 1b-c. Upon

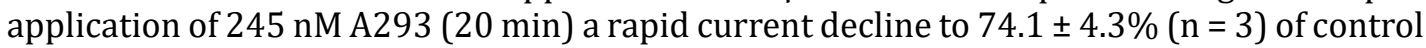
currents was observed (Fig. 1d) and after increasing the blocker concentration to $10 \mu \mathrm{M}(20$ $\mathrm{min})$, relative TASK-1 currents remaining were only $19.3 \pm 7.7 \%(\mathrm{n}=3)$.

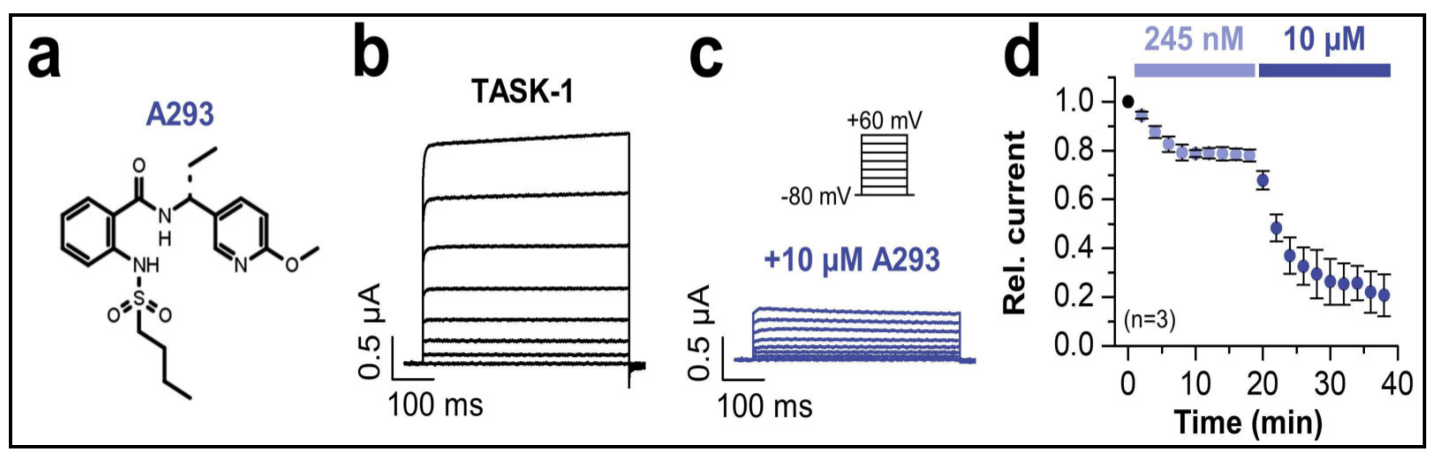

Fig. 1. TASK-1 channel blockade by A293. (a) Chemical structure of the aromatic carbonamide A293. (bc) Human TASK-1 channels were heterologously expressed in Xenopus laevis oocytes. Families of TASK-1 current traces under control conditions (b) and after administration of $10 \mu \mathrm{M} \mathrm{A293}$ (c). The voltage protocol is depicted as inset. (d) Time course of TASK-1 blockade by $240 \mathrm{nM}$ or $10 \mu \mathrm{M}$ of A293 (n = 3). Data are displayed as mean \pm S.E.M. 


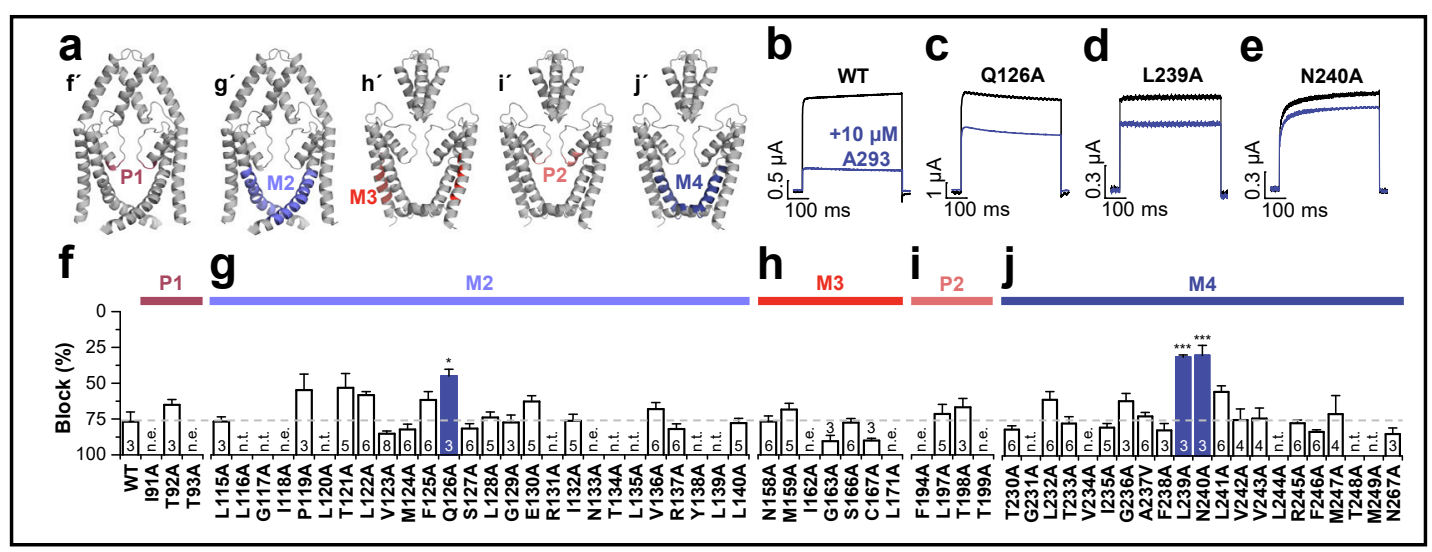

Fig. 2. Alanine-scan to identify the A293 binding site in TASK-1. (a) Amino acids or regions subjected to alanine scanning mutagenesis are displayed in different colors for the different domains, $\mathrm{f}^{\prime}$ for the P1 (pore forming loop 1) pore signature sequence, $g^{\prime}$ for the M2 (transmembrane domain 2) segment, $h^{\prime}$ for the M3 (transmembrane domain 3) segment, $i^{\prime}$ for the P2 (pore forming loop 2) pore signature sequence and $j^{\prime}$ for the M4 (transmembrane domain 4) segment. A TASK-1 homodimer consisting of only M1, P1 and M2 domains where M3, P2 and M4 domains are omitted for clarity is displayed in f' and g'. Homodimers of TASK-1 channels consisting of only M3, P2 and M4 domains where M1, P1 and M2 domains have been omitted are displayed in h'-j'. (b-e) Representative currents of TASK-1 WT and mutant channels before (black) and after the application of $10 \mu \mathrm{M}$ A293 (blue). (f-j) Inhibition of TASK-1 pore mutant channels by $10 \mu \mathrm{M}$ A293 is displayed, compared to TASK-1 WT channels (dashed line). Data are given as mean \pm S.E.M.. ${ }^{*} \mathrm{p}<0.05,{ }^{* * *} \mathrm{p}<0.001$.

\section{Identification of the A293 binding site in TASK-1}

In previous studies TASK-1 and TASK-3 ( $\left.\mathrm{K}_{2 \mathrm{P}} 9.1\right)$ inhibitors were identified to bind at the intracellular central channel cavity [25, 31]. TASK-1 amino acids potentially involved in A293 binding were identified in silico using a TASK-1 homology model, based on the crystal structure of human TWIK-1 $\left(\mathrm{K}_{2 \mathrm{P}} 1.1\right)$, TREK-1 $\left(\mathrm{K}_{2 \mathrm{p}} 2.1\right)$ and TRAAK $\left(\mathrm{K}_{2 \mathrm{P}} 4.1\right)$ channels. Therefore, after visualization of the three dimensional channel structure, a set of 45 amino acids that line the intracellular site of the channel pore were individually mutated to alanine and one alanine residues was changed to valine (Fig. 2a). As observed previously in potassium channels with other domain topologies (for example rKv1.2 and KirBac1.1) these pore lining residues are predominantly located at the transmembrane segments following the selectivity filter [32, 33], corresponding to the M2 and M4 transmembrane domains of TASK-1 [25].

While TASK-1 WT channels were blocked by $10 \mu \mathrm{M}$ A293 by $80.7 \pm 7.7 \%(\mathrm{n}=3)$ (Fig. $2 \mathrm{~b}$ and $f$ ), we identified three residues that showed significantly reduced blocking efficiencies (Fig. 2b-e). In the M2 segment (Fig. 2a and g) we found that TASK-1 Q126A mutant channels were only blocked by $45.1 \pm 4.8 \%(p=0.011, n=3$, Fig. 2c). In the M4 segment (Fig. 2a and j) we found reduced A293 inhibition for L239A and N240A. For TASK-1 L239A channels, A293 effects were reduced with $31.5 \pm 1.5 \%(p=0.0023, n=3$, Fig. $2 d$ and $j)$ and introduction of the N240A mutation yielded channels with a reduction in A293 blockade of $30.3 \pm 7.0 \%$ ( $p=0.050, n=3$, Fig. $2 \mathrm{e}$ and $\mathrm{j}$ ). Recording the $\mathrm{IC}_{50}$ values for the mutations that we have identified in our alanine-scanning approach, we observed a 42 -fold change in $\mathrm{IC}_{50}$ for Q126A, a 108-fold for L239A and a 78-fold for N240, respectively (Supplementary Table 1 - for all supplemental material see www.cellphysiolbiochem.com).

\section{Q126A, L239A and N240A are part of the A293 binding site}

To further support the results of the alanine-scanning mutagenesis, alanine mutations identified to contribute to the A293 drug binding site were introduced as double mutations in single TASK-1 constructs. Combination of the Q126A/L239A mutations almost completely abolished inhibition by A293 ( $\mathrm{n}=4$, Fig. 3b and e). In a similar manner, TASK-1 Q126A/ N240A double mutants displayed only a minor A293-dependent inhibition $(n=5$, Fig. $3 \mathrm{c}$ and e), while the TASK-1 L239A/N240A showed virtually no A293-dependent block at all ( $\mathrm{n}=$ 


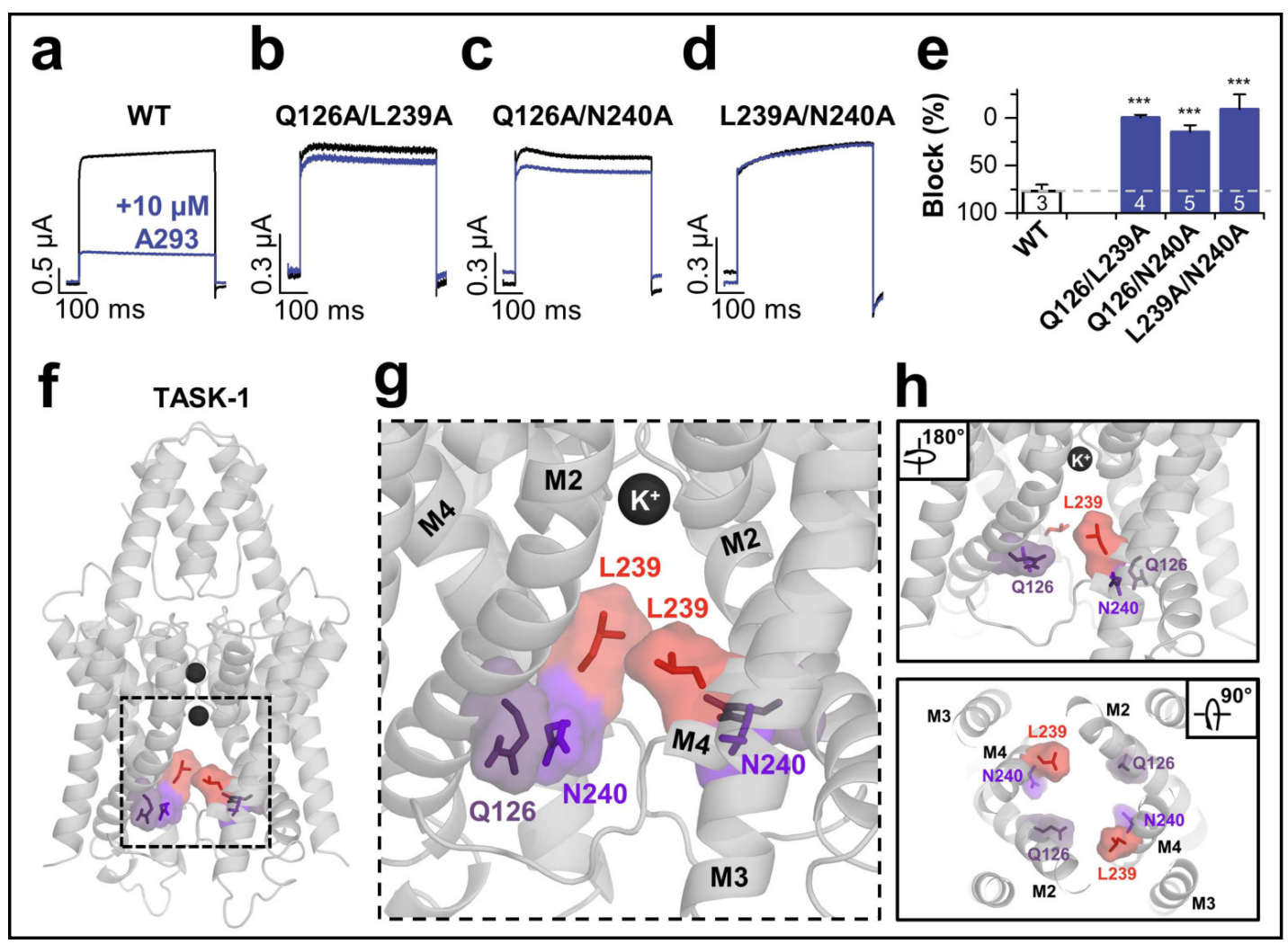

Fig. 3. Q126A, L239A and N240A are part of the A293 binding site: illustration in a TASK-1 pore homology model. (a-e) TASK-1 channels harboring combined mutations of the A293 binding site (double mutations with Q126A, L239A or N240A) show strongly reduced A293 sensitivity. (a-d) Representative current traces under control conditions (black) and after superfusion with $10 \mu \mathrm{M}$ A293 (blue). (e) Analyses of the inhibition of Q126A/L239A, Q126A/N240A and L239A/N240A double mutants by $10 \mu$ M A293. Data are provided as mean \pm S.E.M. ${ }^{* * *} \mathrm{p}<0.001$ vs. WT. (f-g) TWIK-1-based TASK-1 homology model illustrating the A293 binding site at the lower end of the central cavity. (f) Overview illustrating the location of Q126A, L239A and N240A in TASK-1 and (g) zoom into the central cavity. (f-g) Note that the view into the central cavity is possible through the large lateral side fenestrations. (h) Lateral view as in (g) but from the other side of the channel. The lower panel illustrates the view from inside the cell into the central cavity.

5, Fig. 3d and e). A TWIK-1-based TASK-1 homology model reveals that Q126A, L239A and $\mathrm{N} 240 \mathrm{~A}$ are located at approximately the same height at the lower intracellular end of the central channel cavity (Fig. 3f-h). Note that the unrestricted view into the central cavity is possible through the large lateral side fenestrations (LF; Fig. 3f-g).

\section{A293 binds to the lower central cavity at the lateral entry to the LF}

Next we in silico docked A293 into a TWIK-1-based TASK-1 homology model (Fig. 4). We have obtained 100 docking solutions, which include five poses for twenty different conformers (see Methods). Overall we only obtained two significant clusters with 19 and 11 docking solutions, respectively, indicating relatively low variability. Fig. 4a-d illustrate the 30 docking solutions of these two clusters, highlighting that the docking solutions are highly similar. From these docking solutions we have chosen the one that showed (1) interactions with all the residues that we have identified, (2) while it is predicted to prevent $\mathrm{K}^{+}$flux through the central cavity (Supplementary Fig. 1a) and (3) having the lowest energy as the final docking solution. The best docking solution was obtained for an A293 docking pose for which the drug binds at the lower end of the central cavity directly in front of the entry to the LF (Fig. 4a,b,e). A293 binds in the central cavity in close proximity (less than 4 Å distance) to Q126, L239 and N240 identified in our alanine-scanning mutagenesis (Fig. 4e-h). Here 


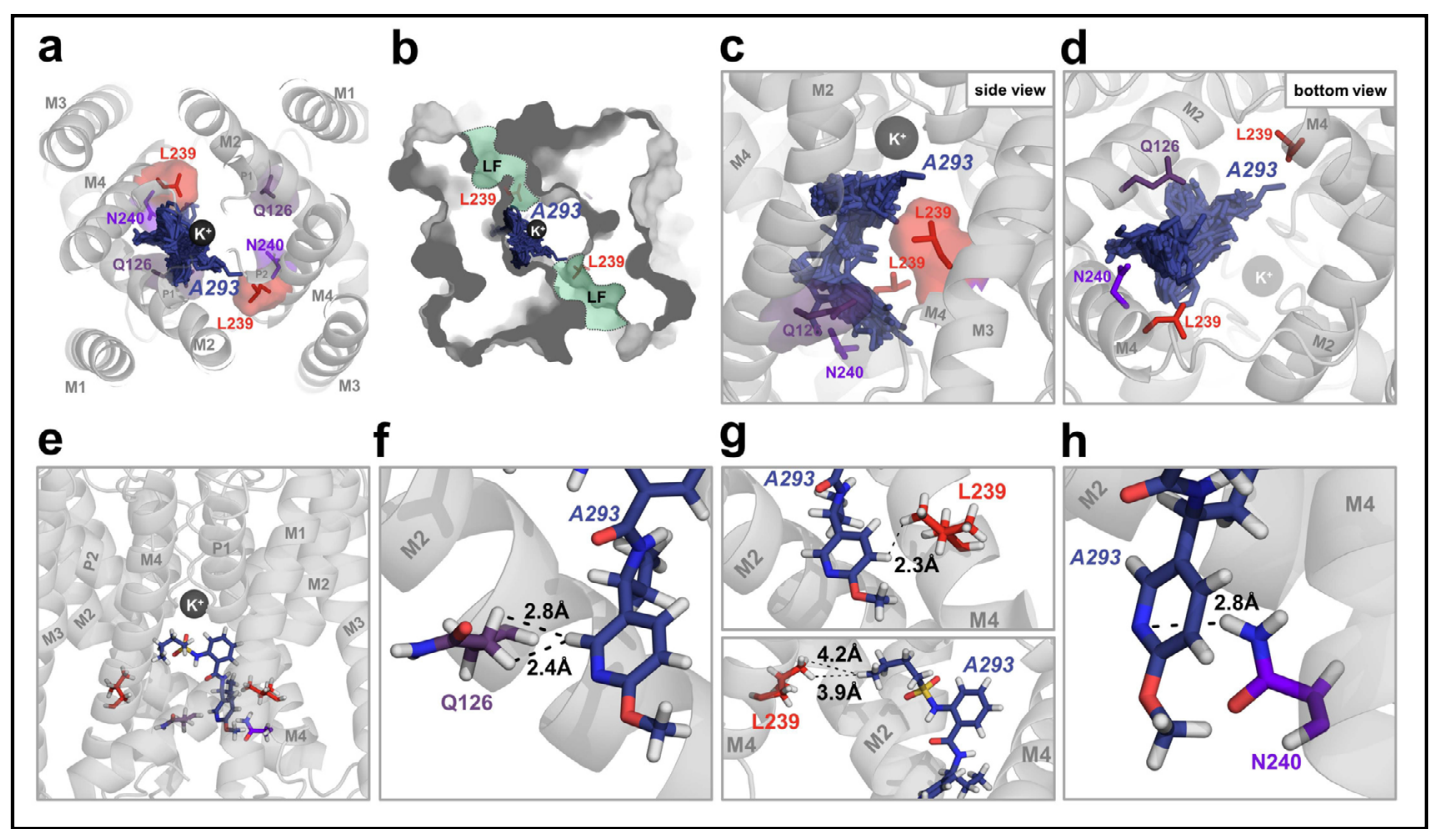

Fig. 4. A293 binds to the lower central cavity at the lateral entry to the side fenestrations. (a-d) A293 binding modes in a TWIK-1-based TASK-1 homology model. Illustrated are all 30 docking solutions of the two significant clusters that we obtained, with 19 and 11 docking solutions, respectively. (a) Bottom view of A293 located in the central cavity in close proximity to Q126, L239 and N240. (b) Similar display as in (a) illustrating the surface area and the side fenestrations (LF). Note that A293 directly binds in front of the entry to the LF, interacting with L239 located in the fenestrations. (c) Zoom into the central cavity illustrating the poses of the two significant clusters and the residues that we identified with the alanine scanning mutagenesis. (d) Similar zoom-in but view from inside the cell. Note that A293 is able to interact with L239 of both fenestrations (distance less than $4 \AA$ Å). (e) Best docking solution of A293 in the TASK-1 homology model and zoom-ins to illustrate the interactions of A293 with (f) Q126, (g) the L239 residue of both subunits and (h) N240.

we identified hydrophobic interactions of the pyridil-group or the butyl side chain of A293 with Q126 or L239 in TASK-1, as well as a H-bond of the pyridil nitrogen atom of A293 to the amide group of N240 (Fig. 4e-h and Supplementary Fig. 2). Note that A293 is located just in front of the LF and is able to interact with both L239 residues located within the pathways of the fenestrations (Fig. 4a,b,e and Supplementary Fig. 2).

\section{Comparing the drug binding site of high affinity and low affinity TASK-1 inhibitors}

In heterologous expression systems, antiarrhythmic drugs block TASK-1 channels when applied in supratherapeutic concentrations [9, 20,21, 22, 26, 34, 35]. To probe whether these low affinity TASK-1 inhibitors and the high affinity blocker A293 share a common drug binding site, Vaughan Williams class I to III antiarrhythmic drugs were tested for their effects on the combined pore alanine mutations. Experiments were conducted using the L239A/ N240A double mutant, as this construct displayed the most robust expression of the double mutants we tested, with an almost complete loss of drug sensitivity at $10 \mu \mathrm{M}$ A293 (Fig. $3 d)$. Upon administration of the class I anti arrhythmic drug propafenone $(100 \mu \mathrm{M})$, TASK-1 WT channels displayed an inhibition of outward potassium currents by $75.4 \pm 7.0 \%(n=3)$ (Fig. 5a), similar as reported in previous studies [9]. The L239A/N240A double mutation however, abolished the inhibitory effects of propafenone at this given concentration (Fig. 5a). After 30 min of perfusion with $100 \mu \mathrm{M}$ propafenone only a $-7.9 \pm 12.8 \%(\mathrm{n}=3)$ increase in outward potassium currents was observed, reflecting a strongly reduced propafenone inhibition ( $p=0.0097, n=3)$. Similar observations were made for the class II antiarrhythmic drug and beta blocking agent carvedilol that was described to exert TASK-1 blocking effects 


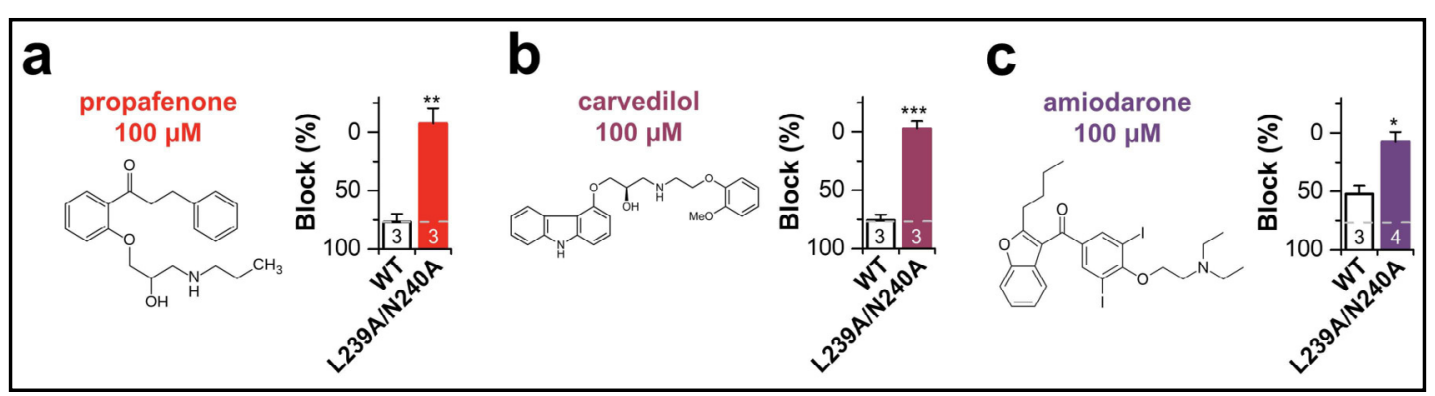

Fig. 5. Overlapping drug binding sites of high and low affinity TASK-1 inhibitors. As previously reported, Vaughan Williams class I (propafenone), II (carvedilol) and III (amiodarone) antiarrhythmic drugs display strong TASK-1 inhibition when applied in supratherapeutic levels $(100 \mu \mathrm{M})$. In TASK-1 channels harboring a L239A/N240A double mutation effects of propafenone (a), carvedilol (b) and amiodarone (c) are virtually abolished. Data are presented as mean plus minus S.E.M.. ${ }^{*} \mathrm{p}<0.05, * * \mathrm{p}<0.01, * * * \mathrm{p}<0.001$.

[21]. While carvedilol $(100 \mu \mathrm{M}$, Fig. 5b) displayed robust inhibition levels on WT TASK-1 channels $(75.7 \pm 4.7 \%, \mathrm{n}=3)$, double mutant TASK-1 L239A/N240A channels were virtually insensitive to carvedilol $(-2.7 \pm 6.5 \%, p=0.00097$, Fig. $5 b)$. Finally, the non-specific potassium channel blocker class III antiarrhythmic drug amiodarone, reduced WT TASK-1 currents by $52.3 \pm 7.3 \%$ (Fig. 5 c) comparable to data from earlier studies [20]. In contrast, amiodaroneinduced blockade of TASK-1 L239A/N240A double mutant channels was strongly reduced ( $\mathrm{p}$ $=0.011$ ) and only $1.1 \pm 7.7 \%$ inhibition remained $(n=3-4$, Fig. $5 c)$.

\section{Discussion}

Safe and effective treatment of AF still remains an unmet clinical need in cardiovascular medicine [18]. To avoid potentially life-threatening proarrhythmic effects in the ventricular myocardium, recent research focuses on the identification of atrial specific drug targets [28]. While other potassium channels that are predominantly expressed in the human atria like Kv1.5 are downregulated in AF-associated electrical remodeling [36] TASK-1 upregulation might potentiate antiarrhythmic effects of TASK-1 inhibition in AF patients $[15,19]$. Experimental Kv1.5 channel inhibitors have recently been described to exert TASK-1 blocking effects $[13,23,25]$ and a number of clinically used antiarrhythmic drugs are known to interact with TASK-1 channels at supratherapeutic plasma levels [37]. In this context we aimed to assess the molecular determinants of the A293 drug binding site in TASK-1 channels. Following in silico identification of TASK-1 inner pore lining amino acid residues, alanine-scanning mutagenesis identified the three residues Q126, L239 and N240 as critical components of the A293 binding site in TASK-1. Combined substitution of two of these residues prevented A293 inhibition of TASK-1 almost completely. In silico docking simulations of A293 to a homology model of TASK-1 predicted an A293 binding pose, with the compound located at the cytosolic end of the central cavity at the opening to the LF. Strikingly, the combined site-directed alanine mutagenesis of L239 and N240 residues also virtually abolished the apparent affinity of TASK-1 to class I - III antiarrhythmic drugs. Thus, high and low affinity antiarrhythmic TASK-1 blockers share overlapping binding sites.

Previously, the drug binding site of the high affinity inhibitor A1899 was revealed for TASK-1, using a comparable alanine-scanning approach [25]. The ten amino acids that contribute to A1899 binding include the L239-N240 region identified in our study. In addition, also the alanine exchange of Q126 was shown to reduce the apparent affinity of A1899. Therefore, drug binding sites of high affinity inhibitors A293 and A1899 display some overlap. While L122 displayed moderate interaction with A293 in our study, I235, V243 and M247 solely contribute to TASK-1 interaction with A1899 and not with A293. 


\section{Cellular Physiology Cell Physiol Biochem 2019;52:1223-1235

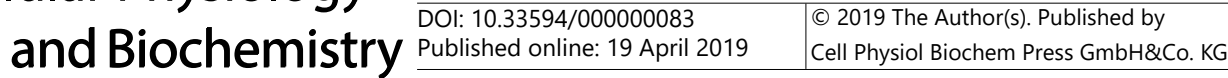 \\ Wiedmann et al.: TASK-1 Drug Binding Site of A293}

Kiper et al. hypothesized that the molecular drug binding sites of TASK-1 and Kv1.5 may share structural similarities as several Kv1.5 inhibitors show high affinity for TASK-1 channels [23]. The authors proposed a structure of three rings encircling the ion conduction pathway (T93 and T199; I118 and I235 as well as L122 and L239). In their model the A293 interaction site would be located at the most intracellular hydrophobic ring leucine consisting of L122 and L239. T93, T199 and I118 could not be investigated in our project due to lack of functionality and I235 was not involved in A293 binding.

To identify the drug interaction site of closely related TASK-3 channels, Chokshi et al. employed a different approach, expressing aspartate mutant channels in fisher rat thyroid cell monolayers [31]. The proposed TASK-3 binding site of doxapram includes residues L122, and L239 contributing to the A1899 binding site in TASK-1 [25]. This points towards conservation of the drug binding site among different members of the TASK subfamily.

When calculating an atomic structural closed state model for the $\mathrm{K}_{2 \mathrm{P}}$ channel TREK-1 in a membrane environment, Treptow and Klein identified the two leucine residues 174 and 289, located in the M2 and M4 domains to delimit the narrowest part of the inner pore and to form a hydrophobic gate [38]. However, when looking at the recently revealed TREK1 crystal structure [39], one can note that it is not L174 but L175 that point towards the central cavity. The TREK-1 leucine residues 175 and 289 align to TASK-1 residues Q126 and L239, identified as part of the A293 drug binding site in this study.

One major difficulty in designing specific TASK-1 blockers, however, remains the high homology of TASK- 1 and TASK- 3 channels. Out of the 46 pore lining amino acids examined in this project, 43 remain conserved among human TASK- 1 and TASK- 3 channels. From the remaining 3 residues L115 and I132 (i.e. V115 and M132, respectively in TASK-3) do not contribute to the A293 drug binding site in TASK-1, while I162 (i.e. V162 in TASK-3) showed no measurable currents. Therefore, all residues identified to contribute to TASK-1 binding of A293 are conserved in TASK-3 channels. This explains the high TASK-3 affinity of A293 reported by Putzke et al. 2007 [13] and our group [15]. Thus, a specific TASK-1 inhibitor that shows no side effects on TASK-3 currents would have to target a different binding site inside the TASK- 1 channel pore. Whether TASK-1 over TASK-3 specificity has to be accomplished for development of a new antiarrhythmic drug remains however uncertain, as TASK-3 channels display in some studies low cardiac abundance [15], while other studies report strong expression of TASK-3 in human atrial cardiomyocytes and heterodimer formation of TASK-1 and TASK-3 [40]. Potential limitations of this study arise from the fact that only functionally active mutant channels were accessible for our investigations. Therefore, contribution of nonfunctional TASK-1 mutant channels I91A, T93A, I118A, N133A, I162A, L171A, F194A, T199A and V234A to the A293 binding site remains unclear. Unfortunately, the alanine mutation at I118 which is one of the most important residues involved in the binding site of A1899, did not express in the framework of the current study. We have already previously noted [25] that the I118A mutant is very poorly expressing. However, consistent with the previously identified role of I118 in drug binding, this residue might be also relevant for the interaction with A293, as it is within less than $4 \AA$ to the butyl group of A293 (Supplementary Fig. 1b). Furthermore, changes in apparent binding affinity were not directly monitor as it could be performed with for example a radioligand binding assay. However, also binding assays would have their limitations as binding does not necessarily indicate inhibition, meaning that the inhibition can be abolished by a mutation while the drug still binds.

\section{Clinical implications}

Emerging evidence suggests a major role of TASK-1 currents in pathogenesis of AF. Better knowledge of the molecular determinants of different drug binding sites in TASK-1 might finally advance the development of more potent and specific TASK-1 inhibitors. Novel specific TASK-1 blockers would be of great use in basic research and furthermore represent lead structures for development of new antiarrhythmic agents. However, it remains speculative whether differences in the specific drug binding site might result in different antiarrhythmic potency of TASK-1 inhibitors. 


\section{Cellular Physiology Cell Physiol Biochem 2019;52:1223-1235

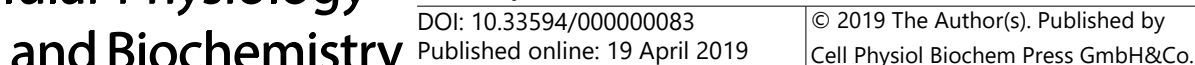 \\ Wiedmann et al.: TASK-1 Drug Binding Site of A293}

\section{Conclusion}

In conclusion, we have mapped the residues in the central cavity of TASK-1 that interact with A293 and mediate the inhibition of TASK-1. Furthermore, substitution of these residues with alanine also reduced the apparent affinity of low affinity inhibitors of the TASK-1 channels like antiarrhythmic drugs. The inferred TASK-1 drug binding site of A293 partially overlaps with the site previously described for A1899 a high affinity inhibitor of TASK-1 [25] and the proposed TASK-3 binding site of doxapram [31]. Further studies will show whether differences in the molecular binding site of TASK-1 blockers affect antiarrhythmic potency of TASK-1 inhibitors.

\section{Abbreviations}

A (alanine); A293 (2-(butylsulfonylamino)-N-[(1R)-1-(6-methoxy-3-pyridyl)propyl] benzamide); AF (atrial fibrillation); HF (heart failure); $\mathrm{K}_{2 \mathrm{P}}$ (two-pore-domain); TASK1 ((TWIK)-related acid sensitive $\mathrm{K}^{+}$channel 1); TASK-3 ((TWIK)-related acid sensitive $\mathrm{K}^{+}$ channel 3); TRAAK (TWIK-related arachidonic acid-stimulated $\mathrm{K}^{+}$channel); TREK-1 (TWIKrelated $\mathrm{K}^{+}$channel 1); TWIK-1 (tandem of $\mathrm{P}$ domains in a weak inward rectifying $\mathrm{K}^{+}$channel); WT (wild type).

\section{Acknowledgements}

The excellent technical support of Sabine Höllriegel, Katrin Kupser and Kai Sona is gratefully acknowledged. We thank Stefan Kallenberger for critical comments and helpful discussion. This study was supported in part by research grants from the University of Heidelberg, Faculty of Medicine (Rahel Goitein-Straus Scholarship and Olympia-Morata Scholarship to C.S.), from the DZHK (German Center for Cardiovascular Research; Excellence Grant to C.S.), from the German Cardiac Society (DGK Research Scholarship to F.W., OttoHess Fellowship to F.W.), from the German Heart Foundation /German Foundation of Heart Research (F/41/15 to C.S., Kaltenbach Scholarship to A.R. and F.W.), from a Fondecyt Grant (1191133 to W.G.), from the Millennium Nucleus of Ion Channels Associated Diseases (MiNICAD) Chile to W.G. and from the Deutsche Forschungsgemeinschaft (German Research Foundation; SCHM 3358/1-1 to C.S. and DE1482-4/1 to N.D.).

F.W., C.S., W.G. and N.D conceived and planned the experiments. F.W., A.R., S.R., M.W., M.K., P.A. and M.B. carried out the experiments. F.W., A.K.K., S.R., A.R., W.W., W.G., N.D. and C.S. contributed to the interpretation of the results. A.K.K. and N.D. visualized the data. F.W., C.S., AK.K. and N.D. wrote the manuscript. H.K., N.D. and C.S. supervised the project. All authors provided critical feedback and helped shape the research, analysis and manuscript.

\section{Disclosure Statement}

The authors declare that they have no conflict of interests. 


\section{Cellular Physiology Cell Physiol Biochem 2019;52:1223-1235

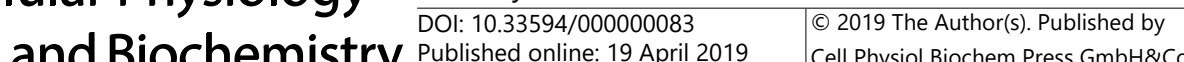 and Biochemistry Published online: 19 April 2019 Cell Physiol Biochem Press GmbH\&Co. KG \\ Wiedmann et al.: TASK-1 Drug Binding Site of A293}

\section{References}

1 Goldstein SAN, Bayliss DA, Kim D, Lesage F, Plant LD, Rajan S: International Union of Pharmacology. LV. Nomenclature and molecular relationships of two-P potassium channels. Pharmacol Rev 2005;57:527540.

- 2 Goldstein SAN, Bockenhauer D, O’Kelly I, Zilberberg N: Potassium leak channels and the KCNK family twoP-domain subunits. Nat Rev Neurosci 2001;2:175-184.

- 3 Feliciangeli S, Chatelain FC, Bichet D, Lesage F: The family of $\mathrm{K}_{2 \mathrm{P}}$ channels: salient structural and functional properties. J Physiol 2015;593:2587-2603.

- 4 Dedman A, Sharif-Naeini R, Folgering JH, Duprat F, Patel A, Honoré E: The mechano-gated $\mathrm{K}_{2 \mathrm{P}}$ channel TREK-1. Eur Biophys J 2009;38:293-303.

- 5 Bayliss DA, Barrett PQ: Emerging roles for two-pore-domain potassium channels and their potential therapeutic impact. Trends Pharmacol Sci 2008;29:566-575.

- 6 Patel AJ, Honoré E: Molecular physiology of oxygen-sensitive potassium channels. Eur Respir J 2001;18:221-227.

- 7 Kim D, Kang D: Role of $\mathrm{K}_{2 \mathrm{P}}$ channels in stimulus-secretion coupling Pflugers Arch 2015;467:1001-1011.

- 8 Lloyd EE, Crossland RF, Phillips SC, Marrelli SP, Reddy AK, Taffet GE, Hartley CJ, Bryan RM Jr: Disruption of $\mathrm{K}_{2 \mathrm{P}} 6.1$ produces vascular dysfunction and hypertension in mice. Hypertension 2011;58:672-678.

- 9 Schmidt C, Wiedmann F, Schweizer PA, Becker R, Katus HA, Thomas D: Class I antiarrhythmic drugs inhibit human cardiac two-pore-domain $\mathrm{K}^{+}\left(\mathrm{K}_{2 \mathrm{P}}\right)$ channels. Eur J Pharmacol 2013;721:237-248.

- 10 Enyedi P, Czirják G Molecular: Background of Leak K+ Currents: Two-Pore Domain Potassium Channels. Physiol Rev 2010;90:559-605.

- 11 Boyle WA, Nerbonne JM: Two functionally distinct 4-aminopyridine-sensitive outward $\mathrm{K}^{+}$currents in rat atrial myocytes. J Gen Physiol 1992;100:1041-1067.

- 12 Backx PH, Marban E: Background potassium current active during the plateau of the action potential in guinea pig ventricular myocytes. Circ Res 1993;72:890-900.

- 13 Putzke C, Wemhöner K, Sachse FB, Rinné S, Schlichthörl G, Li XT, Jaé L, Eckhardt I, Wischmeyer E, Wulf H, Preisig-Müller R, Daut J, Decher N: The acid-sensitive potassium channel TASK-1 in rat cardiac muscle. Cardiovasc Res 2007;75:59-68.

14 Limberg SH, Netter MF, Rolfes C, Rinné S, Schlichthörl G, Zuzarte M, Vassiliou T, Moosdorf R, Wulf H, Daut J, Sachse FB, Decher N: TASK-1 channels may modulate action potential duration of human atrial cardiomyocytes. Cell Physiol Biochem 2011;28:613-624.

- 15 Schmidt C, Wiedmann F, Voigt N, Zhou XB, Heijman J, Lang S, Albert V, Kallenberger S, Ruhparwar A, Szabó G, Kallenbach K, Karck M, Borggrefe M, Biliczki P, Ehrlich JR, Baczkó I, Lugenbiel P, Schweizer PA, Donner BC, Katus HA, et al.: Upregulation of $\mathrm{K}_{2 \mathrm{p}} 3.1 \mathrm{~K}^{+}$Current Causes Action Potential Shortening in Patients With Chronic Atrial Fibrillation. Circulation 2015;132:82-92.

- 16 Schmidt C, Wiedmann F, Zhou XB, Heijman J, Voigt N, Ratte A, Lang S, Kallenberger SM, Campana C, Weymann A, De Simone R, Szabo G, Ruhparwar A, Kallenbach K, Karck M, Ehrlich JR, Baczkó I, Borggrefe M, Ravens U, Dobrev D, et al.: Inverse remodelling of $\mathrm{K}_{2 \mathrm{P}} 3.1 \mathrm{~K}^{+}$channel expression and action potential duration in left ventricular dysfunction and atrial fibrillation: implications for patient-specific antiarrhythmic drug therapy. Eur Heart J 2017;38:1764-1774.

- 17 January CT, Wann LS, Alpert JS, Calkins H, Cigarroa JE, Cleveland JC Jr, Conti JB, Ellinor PT, Ezekowitz MD, Field ME, Murray KT, Sacco RL, Stevenson WG, Tchou PJ, Tracy CM, Yancy CW; American College of Cardiology/American Heart Association Task Force on Practice Guidelines: 2014 AHA/ACC/HRS guideline for the management of patients with atrial fibrillation: a report of the American College of Cardiology/ American Heart Association Task Force on Practice Guidelines and the Heart Rhythm Society. J Am Coll Cardiol 2014;64:e1-76.

18 Kirchhof P, Benussi S, Kotecha D, Ahlsson A, Atar D, Casadei B, Castella M, Diener HC, Heidbuchel H, Hendriks J, Hindricks G, Manolis AS, Oldgren J, Popescu BA, Schotten U, Van Putte B, Vardas P, Agewall S, Camm J, Baron Esquivias G, et al.: 2016 ESC Guidelines for the management of atrial fibrillation developed in collaboration with EACTS. Eur Heart J 2016;37:2893-2962. 


\section{Cellular Physiology Cell Physiol Biochem 2019;52:1223-1235 \begin{tabular}{l|l|l|}
\hline DOI: 10.33594/000000083 & (O) 2019 The Author(s). Published by \\
\hline
\end{tabular} and Biochemistry Published online: 19 April 2019 Cell Physiol Biochem Press GmbH\&Co. KG \\ Wiedmann et al.: TASK-1 Drug Binding Site of A293}

19 Barth AS, Merk S, Arnoldi E, Zwermann L, Kloos P, Gebauer M, Steinmeyer K, Bleich M, Kääb S, Hinterseer M, Kartmann H, Kreuzer E, Dugas M, Steinbeck G, Nabauer M: Reprogramming of the human atrial transcriptome in permanent atrial fibrillation: expression of a ventricular-like genomic signature. Circ Res 2005;96:1022-1029.

20 Gierten J, Ficker E, Bloehs R, Schweizer PA, Zitron E, Scholz E, Karle C, Katus HA, Thomas D: The human cardiac $\mathrm{K}_{2 \mathrm{P}} 3.1$ (TASK-1) potassium leak channel is a molecular target for the class III antiarrhythmic drug amiodarone. Naunyn Schmiedebergs Arch Pharmacol 2010;381:261-370.

21 Staudacher K, Staudacher I, Ficker E, Seyler C, Gierten J, Kisselbach J, Rahm AK, Trappe K, Schweizer PA, Becker R, Katus HA, Thomas D: Carvedilol targets human $\mathrm{K}_{2 \mathrm{P}} 3.1$ (TASK-1) K+ leak channels. Br J Pharmacol 2011;163:1099-1110.

22 Schmidt C, Wiedmann F, Schweizer PA, Becker R, Katus HA, Thomas D: Novel electrophysiological properties of dronedarone: inhibition of human cardiac two-pore-domain potassium $\left(\mathrm{K}_{2 \mathrm{P}}\right)$ channels. Naunyn Schmiedebergs Arch Pharmacol 2012;385:1003-1016.

23 Kiper AK, Rinné S, Rolfes C, Ramírez D, Seebohm G, Netter MF, González W, Decher N: Kv1.5 blockers preferentially inhibit TASK-1 channels: TASK-1 as a target against atrial fibrillation and obstructive sleep apnea? Pflugers Arch 2015;467:1081-1090.

24 Flaherty DP, Simpson DS, Miller M, Maki BE, Zou B, Shi J, Wu M, McManus OB, Aubé J, Li M, Golden JE: Potent and selective inhibitors of the TASK-1 potassium channel through chemical optimization of a bisamide scaffold. Bioorg Med Chem Lett 2014;24:3968-3973.

25 Streit AK, Netter MF, Kempf F, Walecki M, Rinné S, Bollepalli MK, Preisig-Müller R, Renigunta V, Daut J, Baukrowitz T, Sansom MS, Stansfeld PJ, Decher N: A specific two-pore domain potassium channel blocker defines the structure of the TASK-1 open pore. J Biol Chem 2011;286:13977-13984.

26 Seyler C, Duthil-Straub E, Zitron E, Gierten J, Scholz EP, Fink RH, Karle CA, Becker R, Katus HA, Thomas D: TASK-1 $\left(\mathrm{K}_{2 \mathrm{P}} 3.1\right) \mathrm{K}^{+}$channel inhibition by endothelin-1 is mediated through Rho kinase-dependent phosphorylation. Br J Pharmacol 2012;165:1467-1475.

27 Wiedmann F, Schulte JS, Gomes B, Zafeiriou MP, Ratte A, Rathjens F, Fehrmann E, Scholz B, Voigt N, Müller FU, Thomas D, Katus HA, Schmidt C: Atrial fibrillation and heart failure-associated remodeling of twopore-domain potassium $\left(\mathrm{K}_{2 \mathrm{P}}\right)$ channels in murine disease models: focus on TASK-1. Basic Res Cardiol 2018;113:27.

28 Ramírez D, Arévalo B, Martínez G, Rinné S, Sepúlveda FV, Decher N, González W: Side Fenestrations Provide an "Anchor" for a Stable Binding of A1899 to the Pore of TASK-1 Potassium Channels. Mol Pharm 2017;14:2197-2208.

29 Friesner RA, Banks JL, Murphy RB, Halgren TA, Klicic JJ, Mainz DT, Repasky MP, Knoll EH, Shelley M, Perry JK, Shaw DE, Francis P, Shenkin PS: Glide: A New Approach for Rapid, Accurate Docking and Scoring. 1. Method and Assessment of Docking Accuracy. J Med Chem 2004;47:1739-1749.

30 Watts KS, Dalal P, Murphy RB, Sherman W, Friesner RA, Shelley JC: ConfGen: A Conformational Search Method for Efficient Generation of Bioactive Conformers. J Chem Inf Model 2010;50:534-546.

31 Chokshi RH, Larsen AT, Bhayana B, Cottencorresponding JF: Breathing Stimulant Compounds Inhibit TASK3 Potassium Channel Function Likely by Binding at a Common Site in the Channel Pore. Mol Pharmacol 2015;88:926-934.

32 Kuo A, Gulbis JM, Antcliff JF, Rahman T, Lowe ED, Zimmer J, Cuthbertson J, Ashcroft FM, Ezaki T, Doyle DA: Crystal structure of the potassium channel KirBac1.1 in the closed state. Science 2003;300:1922-1926.

33 Long SB, Tao X, Campbell EB, MacKinnon R: Atomic structure of a voltage-dependent $\mathrm{K}^{+}$channel in a lipid membrane-like environment. Nature 2007;450:376-382.

34 Staudacher I, Illg C, Chai S, Deschenes I, Seehausen S, Gramlich D, Müller ME, Wieder T, Rahm AK, Mayer C, Schweizer PA, Katus HA, Thomas D: Cardiovascular pharmacology of K2P17.1 (TASK-4, TALK-2) two-poredomain $\mathrm{K}^{+}$channels. Naunyn Schmiedebergs Arch Pharmacol 2018;391:1119-1131.

35 Schmidt C, Wiedmann F, Gaubatz AR, Ratte A, Katus HA, Thomas D: New Targets for Old Drugs: Cardiac Glycosides Inhibit Atrial-Specific $\mathrm{K}_{2 \mathrm{P}} 3.1$ (TASK-1) Channels. J Pharmacol Exp Ther 2018;365:614-623.

36 Van Wagoner DR, Pond AL, McCarthy PM, Trimmer JS, Nerbonne JM: Outward K+ current densities and Kv1.5 expression are reduced in chronic human atrial fibrillation. Circ Res 1997;80:772-781.

37 Wiedmann F, Schmidt C, Lugenbiel P, Staudacher I, Rahm AK, Seyler C, Schweizer PA, Katus HA, Thomas D: Therapeutic targeting of two-pore-domain potassium $\left(\mathrm{K}_{2 \mathrm{P}}\right)$ channels in the cardiovascular system. Clin Sci (Lond) 2016;130:643-650. 
Cellular Physiology Cell Physiol Biochem 2019;52:1223-1235

\begin{tabular}{ll|l} 
and Biochemistry & $\begin{array}{l}\text { DOI: } 10.33594 / 000000083 \\
\text { Published online: } 19 \text { April } 2019\end{array}$ & $\begin{array}{l}\text { C } 2019 \text { The Author(s). Published by } \\
\text { Cell Physiol Biochem Press GmbH\&Co. KG }\end{array}$ \\
\cline { 2 - 3 } Wiedmann et al.: TASK-1 Drug Binding Site of A293
\end{tabular}

38 Treptow W, Klein ML: The membrane-bound state of $\mathrm{K}_{2 \mathrm{P}}$ potassium channels. J Am Chem Soc 2010;132:8145-8151.

39 Pike ACW, Dong YY, Tessitore A, Goubin S, Strain-Damerell C, Mukhopadhyay S, Kupinska K, Wang D, Chalk R, Berridge G, Grieben M, Shrestha L, Ang JH, Mackenzie A, Quigley A, Bushell SR, Shintre CA, Faust B, Chu A, Dong L: Crystal structure of human two pore domain potassium ion channel TREK1 $\left(\mathrm{K}_{2 \mathrm{P}} 2.1\right)$. URL: https://www.rcsb.org/structure/4TWK.

- 40 Rinné S, Kiper AK, Schlichthörl G, Dittmann S, Netter MF, Limberg SH, Silbernagel N, Zuzarte M, Moosdorf R, Wulf H, Schulze-Bahr E, Rolfes C, Decher N: TASK-1 and TASK-3 may form heterodimers in human atrial cardiomyocytes. J Mol Cell Cardiol 2015;81:71-80. 